University of Nebraska - Lincoln

DigitalCommons@University of Nebraska - Lincoln

\title{
Photoionization cross sections for ions of the cerium isonuclear sequence
}

\author{
M. Habibi \\ University of Nevada - Reno \\ D. A. Esteves \\ University of Nevada - Reno \\ R. A. Phaneuf \\ University of Nevada - Reno, phaneuf@unr.edu
}

A. L. D. Kilcoyne

Lawrence Berkeley National Laboratory, ALKilcoyne@lbl.gov

A. Aguilar

Lawrence Berkeley National Laboratory

See next page for additional authors

Follow this and additional works at: https://digitalcommons.unl.edu/usdoepub

Part of the Bioresource and Agricultural Engineering Commons

Habibi, M.; Esteves, D. A.; Phaneuf, R. A.; Kilcoyne, A. L. D.; Aguilar, A.; and Cisneros, C., "Photoionization cross sections for ions of the cerium isonuclear sequence" (2009). US Department of Energy Publications. 328.

https://digitalcommons.unl.edu/usdoepub/328

This Article is brought to you for free and open access by the U.S. Department of Energy at DigitalCommons@University of Nebraska - Lincoln. It has been accepted for inclusion in US Department of Energy Publications by an authorized administrator of DigitalCommons@University of Nebraska - Lincoln. 


\section{Authors}

M. Habibi, D. A. Esteves, R. A. Phaneuf, A. L. D. Kilcoyne, A. Aguilar, and C. Cisneros

This article is available at DigitalCommons@University of Nebraska - Lincoln: https://digitalcommons.unl.edu/ 


\title{
Photoionization cross sections for ions of the cerium isonuclear sequence
}

\author{
M. Habibi, D. A. Esteves, and R. A. Phaneuf \\ Department of Physics, University of Nevada, MS 220, Reno, Nevada 89557-0058, USA \\ A. L. D. Kilcoyne and A. Aguilar \\ Advanced Light Source, Lawrence Berkeley National Laboratory, MS 7-100, Berkeley, California 94720-8225, USA \\ C. Cisneros \\ Instituto de Ciencias Físicas, Universidad Nacional Autónoma de México, Apartado Postal 48-3, Cuernavaca, Morelos 62251, Mexico
}

(Received 9 June 2009; published 9 September 2009)

\begin{abstract}
Photoionization cross sections for $\mathrm{Ce}^{q+}(1 \leq q \leq 9)$ ions were measured in the $105-180 \mathrm{eV}$ energy range of the $4 d$ inner-shell giant resonance by merging a mass-to-charge-ratio-selected ion beam with a beam of monochromatized synchrotron radiation. The Cowan atomic structure code was used as an aid to interpret the experimental data. Four Rydberg series for $4 d \rightarrow n f(n \geq 4)$ and $4 d \rightarrow n p(n \geq 6)$ autoionizing excitations were assigned using the quantum-defect theory in the $\mathrm{Ce}^{3+}$ photoionization cross section. The experimental data show the collapse of the $n f$ wave functions $(n \geq 4)$ with increasing ionization stage as outer-shell electrons are stripped from the parent ion. The $n f$ orbital collapse occurs partially for $\mathrm{Ce}^{2+}$ and $\mathrm{Ce}^{3+}$ ions and completely for $\mathrm{Ce}^{4+}$, where these wave functions penetrate the core region of the ion. A strong contribution to the total oscillator strength was observed in multiple photoionization channels for $\mathrm{Ce}^{+}, \mathrm{Ce}^{2+}$, and $\mathrm{Ce}^{3+}$, whereas most of the $4 d$ excitations of the higher charge states decay by ejection of one electron.
\end{abstract}

DOI: 10.1103/PhysRevA.80.033407

PACS number(s): $32.80 . \mathrm{Fb}, 32.80 . \mathrm{Aa}, 32.70 . \mathrm{Cs}$

\section{INTRODUCTION}

The experimental study of the nature and character of atoms and ions has always been a challenge for physicists. Photoionization experiments on multiply-charged ions are important as probes of electronic correlation and relativistic effects along isonuclear and isoelectronic series and provide data to benchmark atomic theory and models in plasma physics and astrophysics. Presented in this paper is an experimental investigation over a wide range of charge states of multiply-charged cerium ions. The focus of this investigation was photoionization processes involving $4 d$ electrons along the isonuclear series of cerium ions. The objective was to provide as complete a picture as possible of the ion chargestate dependences of the observed features in the photon energy range of $4 d$ electron excitation and ionization.

Photoionization data for members of the lanthanide group (atomic numbers $Z$ from 57 to 71 ) are of increasing astrophysical interest in connection with studies of nucleosynthesis and star formation [1] since a considerable number of lines belong to the third spectrum (doubly-charged ions), corresponding to the dominant charge state in hot chemically peculiar stars [2]. Cowley reported in 1976 that cerium is the most abundant lanthanide element in the so-called Ap stars $[2,3]$, which emit strong spectral lines of metallic elements.

Photoionization experiments on several isoelectronic and isonuclear sequences were conducted during the last 30 years to study systematics of electron correlation and relativistic effects in the photon energy region of $4 d$ inner-shell excitation of atoms and atomic ions with different numbers of electrons in their outer shells. Photoionization cross-section measurements for $\mathrm{Ba}, \mathrm{Ba}^{+}$, and $\mathrm{Ba}^{2+}$ ions were performed by Lucatorto et al. at the National Bureau of Standards using the dual laser plasma (DLP) technique [4]. They concluded that most of the $4 d$ absorption oscillator strengths of $\mathrm{Ba}$ and $\mathrm{Ba}^{+}$ are in the continuum, whereas the $\mathrm{Ba}^{2+}$ cross section is dominated by strong discrete transitions. Bizau et al. studied the $\mathrm{Ba}^{q+}(q=2-6)$ and $\mathrm{Xe}^{q+}(q=3-7)$ isonuclear sequences using a merged-beam technique at the SuperACO synchrotron light source in France and the Miyake undulator beamline in Denmark [5-10]. Their measurements indicate a dominance of discrete resonances for $\mathrm{Ba}^{4+}$. O'Sullivan et al. measured the $4 d$ photoabsorption of $\mathrm{I}^{q+}$ ions $(q=0-2)$ using the DLP technique [11], concluding that the dominant features arise from $4 d \rightarrow \varepsilon f$ excitation manifested by a shape resonance in neutral iodine and from $4 d \rightarrow n f$ discrete transitions in $\mathrm{I}^{2+}$. The isonuclear sequence of $\mathrm{Cs}$ through $\mathrm{Cs}^{4+}$ was studied by Cummings et al. using the DLP technique [12]. Absolute photoionization cross-section measurements for $\mathrm{Xe}^{4+}, \mathrm{Xe}^{5+}$, and $\mathrm{Xe}^{6+}$ ions were performed at the Advanced Light Source (ALS) in Berkeley by Aguilar et al. [13] using the ionphoton merged-beams technique in support of the development of an extreme ultraviolet lithography light source at a wavelength of $13.5 \mathrm{~nm}$.

Recently, photoionization of Ce encapsulated within the fullerene molecular ion $\mathrm{C}_{82}{ }^{+}$has been investigated by Müller et al. in the photon energy range of $4 d$ excitations [14]. These measurements indicated a clear signature of excitation of the $4 d$ subshell in both single and double ionizations of the $\mathrm{Ce} @ \mathrm{C}_{82}{ }^{+}$endohedral fullerene molecular ion and verified the predicted +3 valency of the encaged $\mathrm{Ce}$ ion.

\section{EXPERIMENTAL TECHNIQUE}

Relative and absolute photoionization cross-section measurements were performed using the ion-photon-beam end station installed on undulator beamline 10.0.1 of the ALS. Cerium ions were produced by evaporating cerium metallocene $\left[\left(\mathrm{C}_{5} \mathrm{H}_{5}\right)_{3} \mathrm{Ce}\right]$ into the plasma discharge of an electron- 


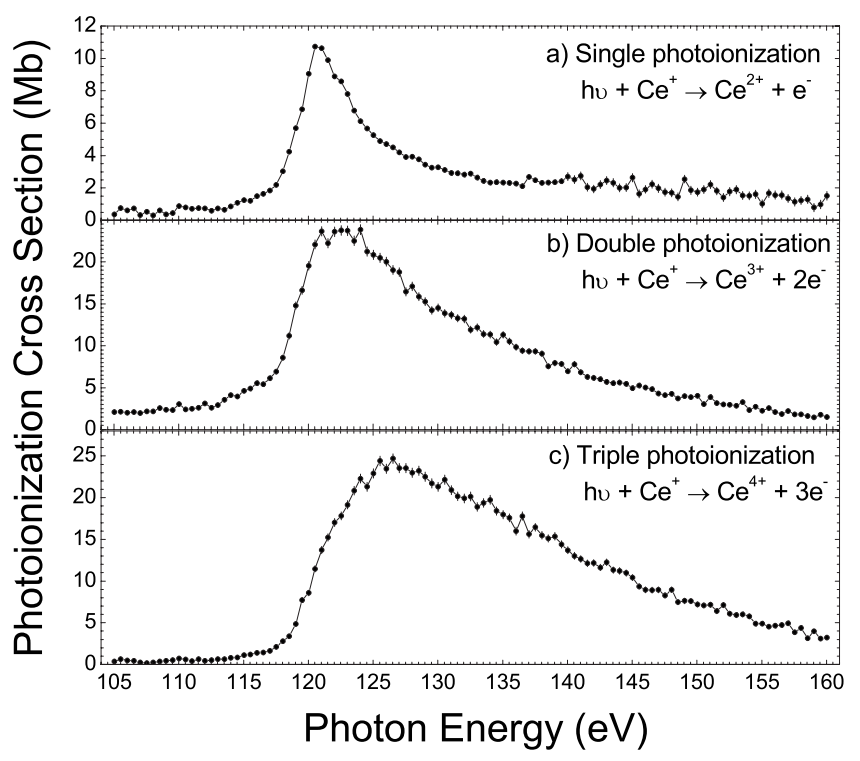

FIG. 1. (a) Single-, (b) double-, and (c) triple-photoionization cross sections of $\mathrm{Ce}^{+}$at a photon energy resolution of $0.1 \mathrm{eV}$.

cyclotron-resonance ion source and an ion beam was extracted by applying a potential of +4 or $+6 \mathrm{kV}$ to the source. A $60^{\circ}$ dipole analyzing magnet selected ions of the desired momentum per charge. Following collimation and focusing, a spherical electrostatic deflector merged the ion beam onto the axis of a counterpropagating beam of monochromatized synchrotron radiation. After interacting with the photon beam over a common path of $1.4 \mathrm{~m}$, the primary and product ion beams were demerged and separated by a $45^{\circ}$ dipole magnet and independently collected by a Faraday cup and a single-particle detector, respectively. For spectroscopic measurements, the photoions were counted as the photon energy was changed in small steps at constant energy resolution. Absolute cross-section measurements were made at discrete photon energies under well-defined conditions, for which two-dimensional spatial intensity profiles of the photon and ion beams were carefully measured at three points within a central interaction region of known length $(29.4 \pm 0.6 \mathrm{~cm})$. The total uncertainty of the cross-section measurements reported here is estimated to be $\pm 23 \%$. Further details concerning the merged-beams apparatus and experimental method may be found in a previous report [15].

\section{RESULTS}

\section{A. Photoionization of $\mathrm{Ce}^{+}$}

Figure 1 presents the measured cross sections for single, double, and triple photoionization of $\mathrm{Ce}^{+}$. A broad asymmetric resonance dominates the cross sections in the energy range where $4 d$ excitations are expected and extends to the $4 d$ continuum. These features are attributed to $4 d \rightarrow \varepsilon f$ transitions. The associated oscillator strengths determined by integrating the cross sections are presented in Table I. Within the experimental uncertainty, a total of 10.8 is consistent with the value of 10 for the $4 d$ subshell predicted by the Thomas-Reiche-Kuhn sum rule.
TABLE I. Oscillator strengths $(f)$ for single, double, and triple photoionization of La-like $\mathrm{Ce}^{+}$.

\begin{tabular}{lccc}
\hline \hline Final charge state & $\begin{array}{c}\text { Energy range } \\
(\mathrm{eV})\end{array}$ & $f$ & $\begin{array}{c}\text { Partial contribution } \\
(\%)\end{array}$ \\
\hline 2 & $105-160$ & 1.55 & 14.2 \\
3 & $105-160$ & 4.18 & 38.6 \\
4 & $105-160$ & 5.10 & 47.2 \\
Total & $105-160$ & 10.8 & 100 \\
\hline \hline
\end{tabular}

Structure attributed to $4 d \rightarrow n p(n \geq 6)$ autoionizing excitations is evident in the single-photoionization cross section [Fig. 1(a)] above $135 \mathrm{eV}$ with a small contribution to the total oscillator strength. So-called "satellite" transitions are likely present above the $4 d$ ionization threshold corresponding to $4 d$ ionization with excitation of one $4 f$ or $5 d$ electron or two $5 d$ electrons $[7,16]$. However, their contribution to the total oscillator strength is very small.

Double photoionization [Fig. 1(b)] is attributed mostly to the $4 d \rightarrow \varepsilon f$ ionization followed by single autoionization competing with multiple autoionization channels. A possible scheme for this process is

$$
\begin{aligned}
\gamma+\mathrm{Ce}^{+}\left(4 d^{10} 4 f 5 d^{2}\right) \rightarrow & \mathrm{Ce}^{2+(*)}\left(4 d^{9} 4 f 5 d^{2}\right)+e^{-}(\varepsilon) \\
& \hookrightarrow \mathrm{Ce}^{3+}\left(4 d^{10} 5 d\right)+2 e^{-} .
\end{aligned}
$$

The weak resonant transitions present in the cross section below the $4 d$ ionization threshold could be due to $4 d \rightarrow n l$ ( $n \geq 6$ and $l=1$ or $n \geq 4$ and $l=3$ ) excitations decaying by sequential ejection of two Auger electrons,

$$
\begin{aligned}
\gamma+\mathrm{Ce}^{+}\left(4 d^{10} 5 p^{6} 4 f 5 d^{2}\right) \rightarrow & \mathrm{Ce}^{+(* *)}\left(4 d^{9} 5 p^{6} 4 f 5 d^{2} n l\right) \\
& \hookrightarrow \mathrm{Ce}^{2+(*)}\left(4 d^{10} 5 p^{5} 4 f 5 d^{2}\right)+e^{-} \\
& \hookrightarrow \mathrm{Ce}^{3+}\left(4 d^{10} 5 p^{6} 4 f\right)+2 e^{-} .
\end{aligned}
$$

Triple photoionization [Fig. 1(c)] is attributed mainly to direct $4 d$ photoionization followed by the ejection of two sequential Auger electrons. Two autoionization paths are suggested for this process,

$$
\begin{aligned}
\gamma+\mathrm{Ce}^{+}\left(4 d^{10} 5 p^{6} 4 f 5 d^{2}\right) \rightarrow & \mathrm{Ce}^{2+(* *)}\left(4 d^{9} 5 p^{6} 4 f 5 d^{2}\right)+e^{-}(\varepsilon) \\
& \hookrightarrow \mathrm{Ce}^{3+(*)}\left(4 d^{10} 5 p^{5} 5 d^{2}\right)+2 e^{-} \\
& \hookrightarrow \mathrm{Ce}^{4+}\left(4 d^{10} 5 p^{6}\right)+3 e^{-}
\end{aligned}
$$

or

$$
\begin{aligned}
\gamma+ & \mathrm{Ce}^{+}\left(4 d^{10} 5 s^{2} 5 p^{6} 4 f 5 d^{2}\right) \\
& \rightarrow \mathrm{Ce}^{2+(* *)}\left(4 d^{9} 5 s^{2} 5 p^{6} 4 f 5 d^{2}\right)+e^{-}(\varepsilon) \\
& \hookrightarrow \mathrm{Ce}^{3+(*)}\left(4 d^{10} 5 s 5 p^{6} 5 d^{2}\right)+2 e^{-} \\
& \hookrightarrow \mathrm{Ce}^{4+}\left(4 d^{10} 5 s^{2} 5 p^{5} 5 d\right)+3 e^{-} .
\end{aligned}
$$

The weak resonant structure in the cross section likely arises from excitation of a $4 d$ electron to a higher energy state followed by ejection of three Auger electrons, 


$$
\begin{aligned}
\gamma+ & \mathrm{Ce}^{+}\left(4 d^{10} 5 s^{2} 5 p^{6} 4 f 5 d^{2}\right) \\
& \rightarrow \mathrm{Ce}^{+(* * *)}\left(4 d^{9} 5 s^{2} 5 p^{6} 4 f 5 d^{2} n l\right) \\
& \hookrightarrow \mathrm{Ce}^{2+(* *)}\left(4 d^{10} 5 s 5 p^{6} 4 f 5 d^{2}\right)+e^{-} \\
& \hookrightarrow \mathrm{Ce}^{3+(*)}\left(4 d^{10} 5 s^{2} 5 p^{5} 4 f 5 d\right)+2 e^{-} \\
& \hookrightarrow \mathrm{Ce}^{4+}\left(4 d^{10} 5 s^{2} 5 p^{6}\right)+3 e^{-} .
\end{aligned}
$$

\section{B. Photoionization of $\mathrm{Ce}^{2+}$}

Measured cross sections for single, double, and triple photoionization of $\mathrm{Ce}^{2+}$ are presented in Fig. 2 along with their associated oscillator strengths in Table II.

Discrete resonances due to autoionizing excitations of the $4 d$ inner shell to $4 f$ and $n p(n \geq 6)$ outer shells are evident in the single-photoionization cross section below $127.5 \mathrm{eV}$. Direct photoionization dominates above $127.5 \mathrm{eV}$, constituting $38 \%$ of the oscillator strength for this channel. Identification of $4 d \rightarrow n p$ Rydberg series was not possible due to the small oscillator strengths of these transitions compared to those of $4 d \rightarrow 4 f$ transitions.

A broad asymmetric shape resonance dominates the double-photoionization cross section where $4 d$ excitations are expected and extends to the $4 d$ continuum, similar to that observed in photoionization of $\mathrm{Ce}^{+}$. This is attributed to $4 d$ $\rightarrow \varepsilon f$ direct photoionization accompanied by double autoionization. Resonant photoionization attributed to $4 d \rightarrow 4 f$ excitations followed by autoionization is evident in the cross section below $127 \mathrm{eV}$. Features due to $4 d \rightarrow n p$ autoionizing excitations may also be present but calculations indicated their contribution to the total oscillator strength to be relatively small.

The energy onset of the triple-photoionization cross section indicates a threshold of $123.5 \pm 1.3 \mathrm{eV}$, which compares to $122.5 \mathrm{eV}$ tabulated by NIST [17]. Possible weak resonant features likely result from excitation of the $4 d$ electrons to higher energy states followed by emission of three Auger electrons. The oscillator strengths in Table II indicate the

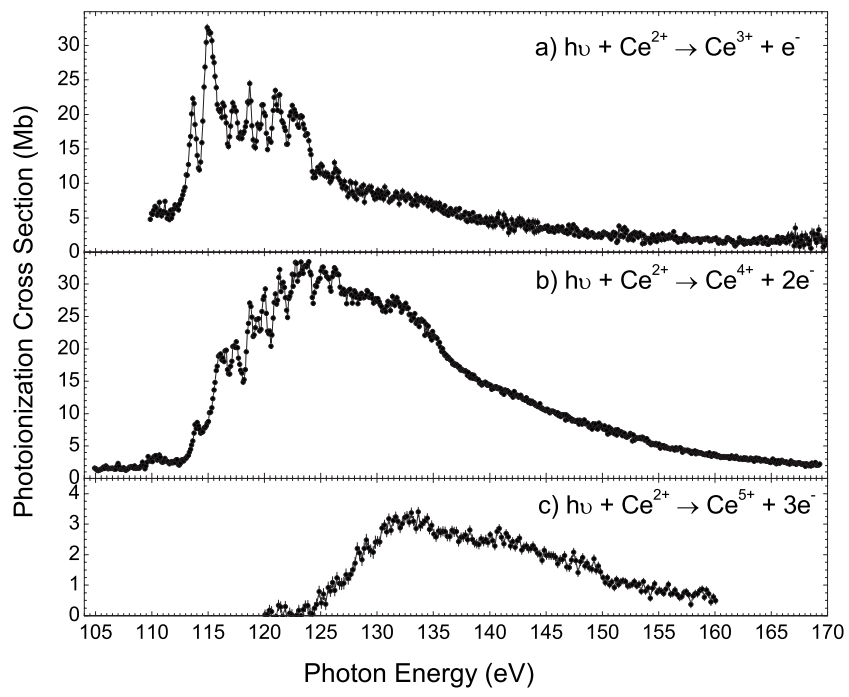

FIG. 2. (a) Single-, (b) double-, and (c) triple-photoionization cross sections of $\mathrm{Ce}^{2+}$ at a photon energy resolution of $0.1 \mathrm{eV}$. dominance of double photoionization, which constitutes more than half of the total photoionization oscillator strength of $\mathrm{Ce}^{2+}$ in this energy range. Since Auger decay of the $4 d$ vacancy states dominates compared to radiative decay, the total oscillator strength in Table II is expected to correspond to the total for the $4 d$ subshell. The measured value greater than 10 (the number of $4 d$ electrons) is not surprising since direct photoionization of $5 s, 5 p$, and $4 f$ electrons is also possible in this energy range.

\section{Photoionization of $\mathrm{Ce}^{3+}$}

Single-photoionization cross section for $\mathrm{Ce}^{3+}$, presented in Fig. 3(a), is dominated by $4 d \rightarrow n f$ and $4 d \rightarrow n p$ autoionizing excitations. Two Rydberg series resulting from transitions of $4 d$ electrons to excited $n f$ and $n p$ orbitals were identified and assigned. Figure 4 presents two fits of the experimental resonance energies as functions of the principal quantum number by use of the quantum-defect form of the Rydberg formula,

$$
E_{n}=E_{\text {limit }}-\frac{R_{\mathrm{Ce}}\left(Z-N_{c}\right)^{2}}{\left(n-\delta_{n}\right)^{2}} .
$$

$E_{n}$ is the transition energy, $E_{\text {limit }}$ is the ionization potential of the electron being excited to the corresponding final state $(n=\infty), Z$ is the nuclear charge, and $N_{c}$ is the number of core electrons. The dimensionless quantum-defect parameter $\delta_{n}$ is a measure of the departure of the energy level $E_{n}$ from a pure hydrogenic value $\left(\delta_{n}=0\right)$. The Rydberg constant $R_{\mathrm{Ce}}$ for cerium ions is given by

$$
R_{\mathrm{Ce}}=\frac{\mu e^{4}}{4 \pi \hbar c},
$$

where $\mu=\left(1 / m_{e}+1 / M_{\mathrm{Ce}}\right)^{-1}$ is the reduced mass of the electron-nucleus system of the cerium ion, $e$ and $m_{e}$ are the charge and the mass of the electron, respectively, $\hbar$ is the reduced Planck constant, and $M_{\mathrm{Ce}}$ is the mass of the cerium nucleus. In the present analysis, $\delta_{n}$ was replaced by a mean quantum-defect parameter $\delta$ for each Rydberg series.

The first Rydberg series is attributed to autoionizing excitations of $\mathrm{Ce}^{3+}$ in the ground state to $4 d^{9} 5 s^{2} 5 p^{6} 4 f^{1}\left({ }^{1} F\right) n f^{1}{ }^{2} P_{3 / 2}(n \geq 4)$ states converging to the $4 d^{9} 5 s^{2} 5 p^{6} 4 f^{1}{ }^{1} F$ limit of $\mathrm{Ce}^{4+}$. The calculated energy limit $\left(E_{\text {limit }}\right)$ for this series is $150.21 \mathrm{eV}$ using the Cowan code [18-20], compared to the fit value of $150.48 \mathrm{eV}$ using the quantum-defect theory. The second Rydberg series originates from $4 d$ excitations of the ${ }^{2} F_{7 / 2}^{o}$ metastable state to

TABLE II. Oscillator strengths $(f)$ for single, double, and triple photoionization of Ba-like $\mathrm{Ce}^{2+}$.

\begin{tabular}{lccc}
\hline \hline Final charge state & $\begin{array}{c}\text { Energy range } \\
(\mathrm{eV})\end{array}$ & $f$ & $\begin{array}{c}\text { Partial contribution } \\
(\%)\end{array}$ \\
\hline 3 & $110-173$ & 4.04 & 33.4 \\
4 & $105-169$ & 7.47 & 61.7 \\
5 & $120-160$ & 0.59 & 4.9 \\
Total & & 12.10 & 100.0 \\
\hline \hline
\end{tabular}




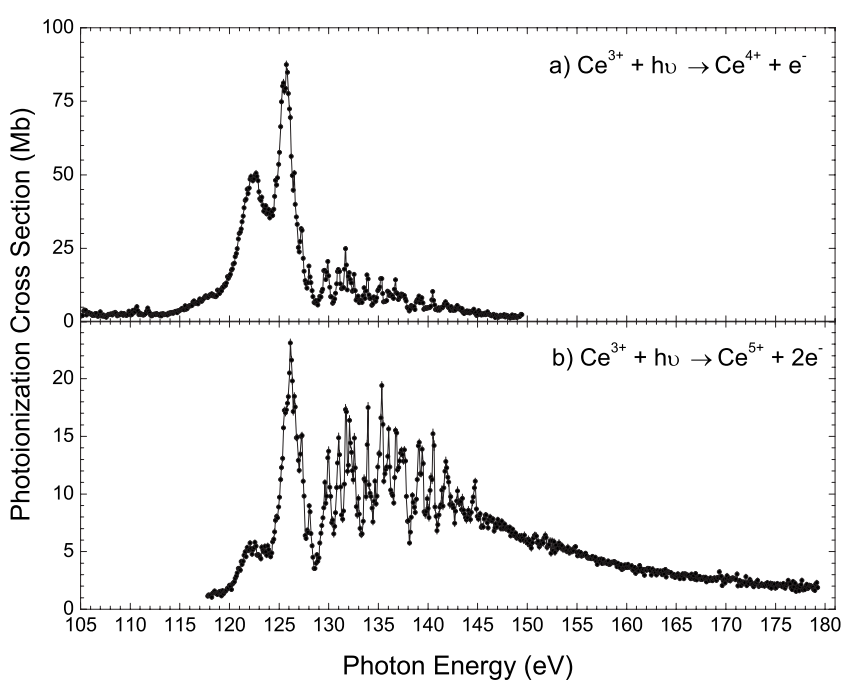

FIG. 3. (a) Single- and (b) double-photoionization cross sections of $\mathrm{Ce}^{3+}$ at a photon energy resolution of $0.1 \mathrm{eV}$.

$4 d^{9} 5 s^{2} 5 p^{6} 4 f^{1}\left({ }^{3} H\right) n p^{1{ }^{4}} G_{9 / 2}(n \geq 6)$ states converging to the $4 d^{9} 5 s^{2} 5 p^{6} 4 f^{13} H$ limit of $\mathrm{Ce}^{4+}$. The energy limit of 143.33 $\mathrm{eV}$ of this series is in good agreement with the Cowan code calculated energy of $143.34 \mathrm{eV}$ [18].

Figure 3(b) shows the double-photoionization crosssection measurements for $\mathrm{Ce}^{3+}$ from 105 to $180 \mathrm{eV}$ photon energy at $0.1 \mathrm{eV}$ resolution. Direct photoionization accompanied by autoionization dominates the cross section above 150 $\mathrm{eV}$. This may be represented by the following process:

$$
\begin{aligned}
\gamma+\mathrm{Ce}^{3+}\left(4 d^{10} 5 s^{2} 5 p^{6} 4 f^{1}\right) \rightarrow & \mathrm{Ce}^{4+(*)}\left(4 d^{9} 5 s^{2} 5 p^{6} 4 f^{1}\right)+e^{-} \\
& \hookrightarrow \mathrm{Ce}^{5+}\left(4 d^{10} 5 s^{2} 5 p^{5}\right)+2 e^{-} .
\end{aligned}
$$

Resonant photoionization cross-section features observed below $150 \mathrm{eV}$ originate from $4 d$ excitations to higher energy $n f$ and $n p$ orbitals accompanied by emission of two Auger electrons. Two Rydberg series were assigned in the doublephotoionization cross section of $\mathrm{Ce}^{3+}$, both originating from the ground state. The corresponding energies and fits are in-
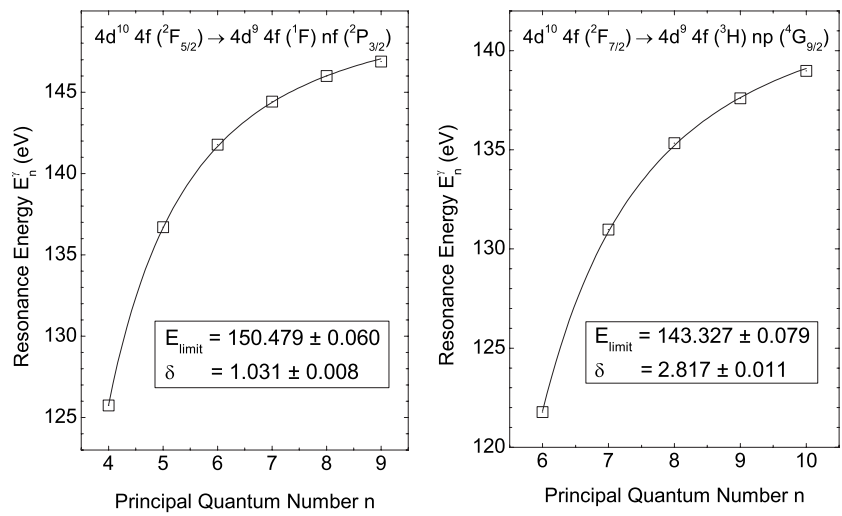

FIG. 4. Rydberg fits for the $4 d^{10} 4 f^{1} n f^{12} P_{3 / 2}$ and $4 d^{10} 4 f^{1} n p^{14} G_{9 / 2}$ series originating from the ${ }^{2} F_{5 / 2}$ ground state and the ${ }^{2} F_{7 / 2}$ metastable state, respectively, in the singlephotoionization cross section of $\mathrm{Ce}^{3+}$. The series limit $E_{\text {limit }}$ and quantum-defect parameter $\delta$ are given for each series.
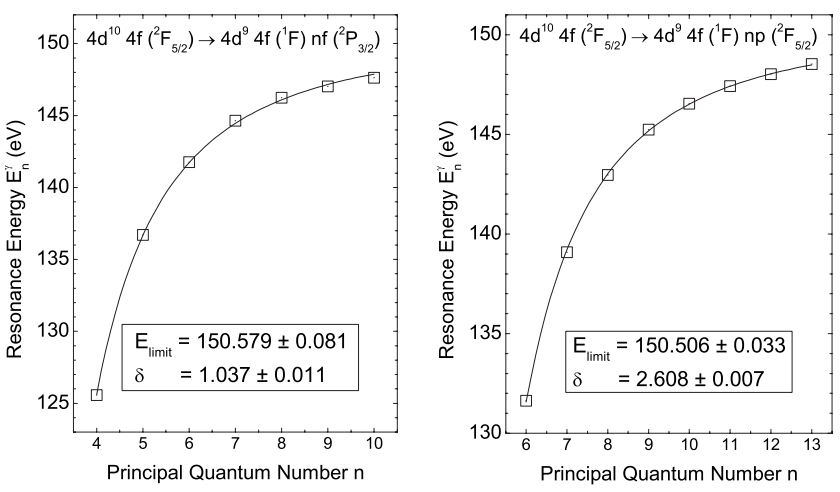

FIG. 5. Rydberg fits for the $4 d^{10} 4 f^{1} n f^{12} P_{3 / 2}$ and $4 d^{10} 4 f^{1} n p^{12} F_{5 / 2}$ series originating from the ${ }^{2} F_{5 / 2}$ ground state in the double-photoionization cross section of $\mathrm{Ce}^{3+}$.

dicated in Fig. 5 The first series results from excitation to the $4 d^{9} 5 s^{2} 5 p^{6} 4 f^{1}\left({ }^{1} F\right) n f^{1}{ }^{2} P_{3 / 2}$ final state, which is identical to the first series observed in the single-photoionization cross section. An increase in the double-to-single-photoionization cross-section ratio for the higher members of this series at increasing photon energy indicates the competition between different autoionizing channels. The second Rydberg series arises from excitations to $4 d^{9} 5 s^{2} 5 p^{6} 4 f^{1}\left({ }^{1} F\right) n p^{1}{ }^{2} F_{5 / 2}$ final states. No identifications were made of Rydberg series due to double photoionization from metastable states.

Oscillator strengths determined from the cross sections for single and double photoionization of $\mathrm{Ce}^{3+}$ are 5.03 and 3.64 , respectively. Their sum of 8.67 is consistent with the expected value of 10 within the experimental uncertainty.

\section{Photoionization of $\mathrm{Ce}^{4+}$}

Figure 6 shows the single-photoionization cross section of $\mathrm{Ce}^{4+}$ in the $4 d$ inner-shell excitation region. The structure below $129.6 \mathrm{eV}$ is attributed mostly to $4 d^{10} 5 s^{2} 5 p^{5} 4 f$ $\rightarrow 4 d^{9} 5 s^{2} 5 p^{5} 4 f^{2}$ transitions followed by ejection of an Auger electron. The features are very broad below $126 \mathrm{eV}$. Direct photoionization of the $5 s^{2}$ subshell is also possible in this energy region, although its contribution to the total cross section is relatively small. The strongest resonance at 131.09 $\mathrm{eV}$ is assigned to $4 d^{10} 5 p^{6}{ }^{1} S_{0} \rightarrow 4 d^{9} 5 p^{6}\left(4 f^{2} F\right)^{3} D_{1}$ excitation followed by autoionization. Resonances near and including the strongest feature (129.7-135 eV) are attributed to unresolved fine structure of the final states of the $4 d^{9} 5 p^{6} 4 f$ configuration. The structures at 136.52 and $139.09 \mathrm{eV}$ are assigned to $4 d \rightarrow 6 p$ excitation from the ground state. The first is attributed to the $4 d^{9} 5 p^{6}\left(6 p^{2} P\right)^{1} P_{1}$ final state and the second to $4 d^{9} 5 p^{6}\left(6 p^{2} P\right)^{3} P_{j}$ final states. The measured energy splitting between the ${ }^{1} P_{1}$ and ${ }^{3} P_{1}$ fine states is $2.57 \mathrm{eV}$, which compares to a calculated value of $2.65 \mathrm{eV}$. At this energy resolution, the first resonance appears more symmetric than the second due to the hyperfine structure splittings in the latter, which is evident in measurements performed at higher photon energy resolution (not shown). The structures near 144.6 and $148 \mathrm{eV}$ arise from the transitions $4 d^{10} 5 p^{6}{ }^{1} S_{0} \rightarrow 4 d^{9} 5 p^{6}\left(5 f^{2} F\right)^{3} D_{j} \quad$ and $4 d^{10} 5 p^{6}{ }^{1} S_{0}$ $\rightarrow 4 d^{9} 5 p^{6}\left(5 f^{2} F\right)^{1} P_{1}$, respectively. The measured splitting of 


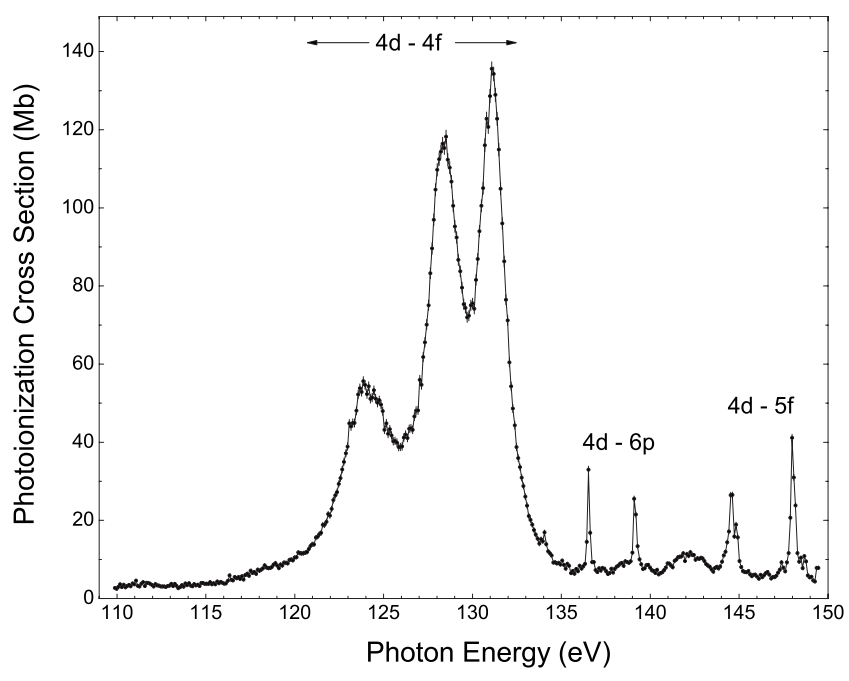

FIG. 6. Single-photoionization cross section of $\mathrm{Ce}^{4+}$ at a photon energy resolution of $0.1 \mathrm{eV}$.

the fine-structure states ${ }^{3} D_{1}$ and ${ }^{1} P_{1}$ is $3.5 \mathrm{eV}$ compared to $3.4 \mathrm{eV}$ in the calculations. Hyperfine structure is observed in the ${ }^{3} D_{1}$ peak. The measured energy splitting between the ${ }^{3} D_{1}$ and ${ }^{3} D_{3}$ states is $0.27 \mathrm{eV}$, compared to a calculated value of $0.42 \mathrm{eV}$. A small resonance near the ${ }^{1} P_{1}$ peak at $148.7 \mathrm{eV}$ is attributed to excitation of $4 d$ electrons from the ground state to $4 d^{9} 5 p^{6}\left(7 p^{2} P\right)^{1} P_{1}$.

The total oscillator strength determined from the measurements in this energy range is 10.32 , consistent with the expected value of 10 for the $4 d$ subshell.

\section{E. Photoionization of $\mathrm{Ce}^{5+}$}

Figure 7(a) shows the absolute photoionization cross section measurements for $\mathrm{Ce}^{5+}$. The total experimental oscillator strength is 10.77 , which is in agreement with the sum-rule value of 10 within the experimental uncertainty. The structure above $131 \mathrm{eV}$ arises mostly from $4 d \rightarrow 4 f$ excitation from the ${ }^{2} P_{3 / 2}$ ground state and from the ${ }^{2} P_{1 / 2}$ metastable

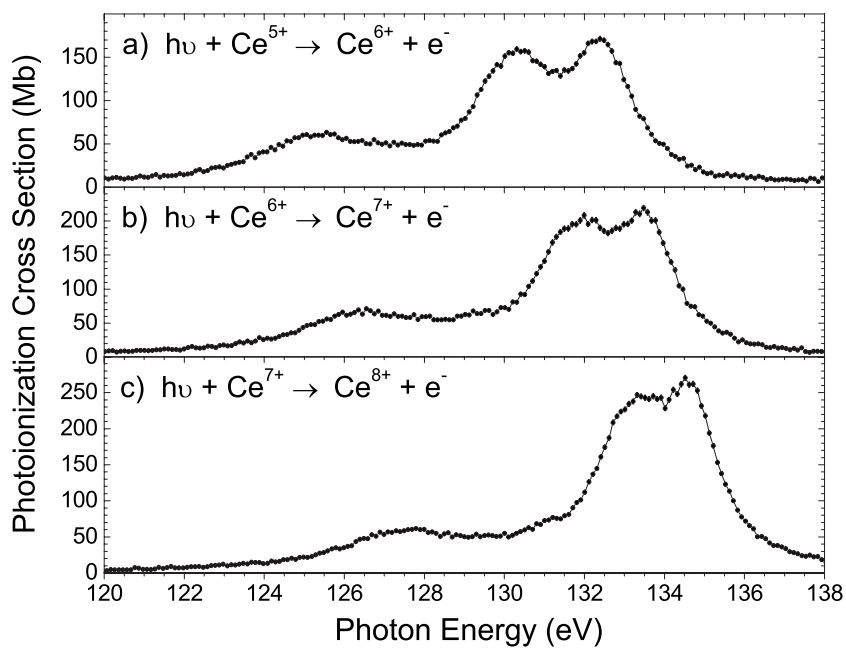

FIG. 7. Single-photoionization cross sections of (a) $\mathrm{Ce}^{5+}$, (b) $\mathrm{Ce}^{6+}$, and (c) $\mathrm{Ce}^{7+}$ at a photon energy resolution of $0.1 \mathrm{eV}$. state, followed by autoionization. Features below $131 \mathrm{eV}$ are attributed to $4 d \rightarrow 4 f$ excitation from the $4 d^{10} 5 s^{2} 5 p^{4} 4 f$ metastable states of $\mathrm{Ce}^{5+}$ followed by autoionization. The measurements suggest that these autoionizing decays occur very rapidly, yielding broad resonances.

\section{F. Photoionization of $\mathrm{Ce}^{6+}$}

Absolute photoionization cross-section measurements for $\mathrm{Ce}^{6+}$ are presented in Fig. 7(b). Above $132.5 \mathrm{eV}$, the measured structures are dominated by $4 d \rightarrow 4 f$ transitions from the ground-state ${ }^{3} P_{2}$ and from the metastable states ${ }^{3} P_{0},{ }^{3} P_{1}$, ${ }^{1} D_{2}$, and ${ }^{1} S_{0}$, followed by autoionization. The structures observed in the range from 115 to $132.5 \mathrm{eV}$ are attributed mainly to $4 d \rightarrow 4 f$ excitation from the $4 d^{10} 5 s^{2} 5 p^{3} 4 f$ metastable states of $\mathrm{Ce}^{6+}$, followed by autoionization. The relative contributions of these metastable states to the total measured photoionization oscillator strength of $\mathrm{Ce}^{6+}(11.4)$ depend on their proportions in the primary ion beam. For photoionization of a pure ground-state $\mathrm{Ce}^{6+}$ ion beam, only the broad resonance peak centered near $133.5 \mathrm{eV}$ would be expected.

\section{G. Photoionization of $\mathrm{Ce}^{7+}$}

Figure 7(c) shows the measured photoionization cross section of $\mathrm{Ce}^{7+}$. The structure observed below $128.5 \mathrm{eV}$ is dominated by autoionizing excitations of $4 d$ electrons from the $4 d^{10} 5 s^{2} 5 p^{2} 4 f$ metastable states to $4 d^{9} 5 s^{2} 5 p^{2} 4 f^{2}$ excited states. Resonances in the energy range of 128.5-134 eV are attributed to $4 d \rightarrow 4 f$ transitions arising from an admixture of the ground state and metastable states. Above $134 \mathrm{eV}$, the structures are assigned to $4 d \rightarrow 4 f$ excitations from the ${ }^{4} S_{3 / 2}$ ground state and ${ }^{2} D_{3 / 2},{ }^{2} D_{5 / 2},{ }^{2} P_{1 / 2}$, and ${ }^{2} P_{3 / 2}$ metastable states. The measured photoionization oscillator strength for $\mathrm{Ce}^{7+}$ is 12.23 , which is consistent with the expected value of 10 within the estimated experimental uncertainty of $\pm 23 \%$.

\section{H. Photoionization of $\mathrm{Ce}^{8+}$}

Small resonant features observed in the photoionization cross section of $\mathrm{Ce}^{8+}$ (Fig. 8) below $127 \mathrm{eV}$ are attributed to $5 s \rightarrow n p(n \geq 8)$ transitions. A small step in the cross section near $125 \mathrm{eV}$ corresponds to the opening of the $5 p$ direct photoionization channel from the ${ }^{3} P_{0}$ ground state. The step near $122 \mathrm{eV}$ corresponds to the photoionization threshold of the ${ }^{3} P_{2}$ metastable state, which lies $3.64 \mathrm{eV}$ above the ground state [18]. However, accurate values of these thresholds could not be determined due to resonances embedded in the cross section in this energy range. Features in the energy range of $127-135.5 \mathrm{eV}$ are attributed to $4 d \rightarrow 4 f$ transitions from an admixture of the ground state and metastable states, followed by autoionization. The features above $135.5 \mathrm{eV}$, including the strongest narrow resonance at $135.96 \mathrm{eV}$, are assigned to autoionizing $4 d \rightarrow 4 f$ excitations from the ${ }^{3} P_{0}$ ground state and the ${ }^{3} P_{1},{ }^{3} P_{2},{ }^{1} D_{2}$, and ${ }^{1} S_{0}$ metastable levels of the ground-state configuration. Contributions of direct $5 \mathrm{~s}$ photoionization from the ground state and metastable states are expected above $138 \mathrm{eV}$ [18]. The integrated experimental oscillator strength in the energy range of these measurements is 12.45 . A value larger than 10 is to be expected in this case 


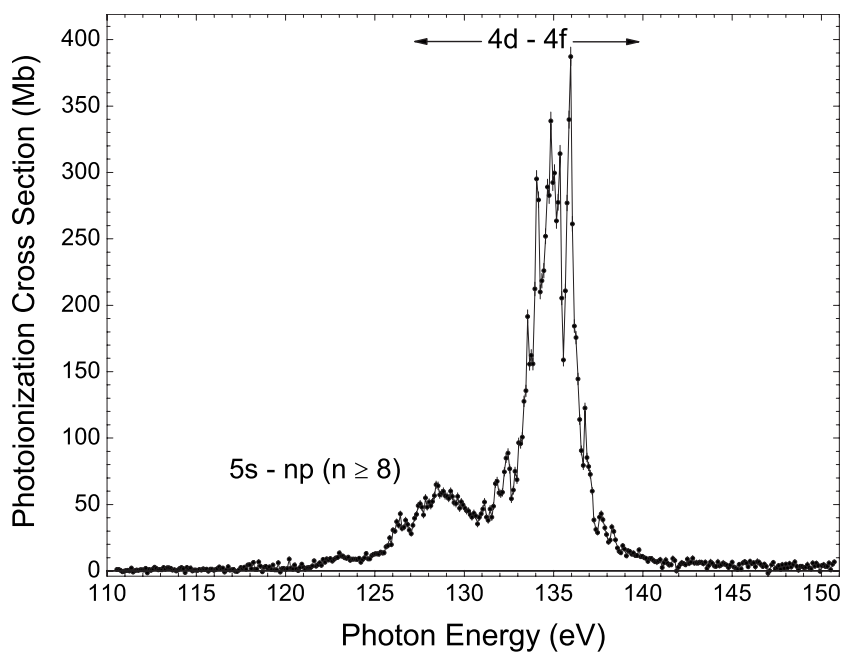

FIG. 8. Single-photoionization cross section of $\mathrm{Ce}^{8+}$ at a photon energy resolution of $0.1 \mathrm{eV}$.

because of direct and resonant contributions to the photoionization cross section from the $5 s$ and $5 p$ subshells in this energy range.

\section{Photoionization of $\mathrm{Ce}^{9+}$}

Photoionization of $\mathrm{Ce}^{9+}$ (Fig. 9) shows little evidence for a direct photoionization threshold from the ground state, which is calculated to occur near $140.83 \mathrm{eV}$ [18]. Therefore, no structure arising due to $4 d$ excitation from the ground state was expected in this energy range. A small step in the cross section near $134.1 \mathrm{eV}$ is attributed to the photoionization threshold of the $4 d^{10} 5 s^{2} 4 f^{12} F_{5 / 2}$ metastable state, which is close to the calculated value of $133.63 \mathrm{eV}$ [18]. The strong resonances above this threshold up to $136.8 \mathrm{eV}$ are mostly attributed to $4 d \rightarrow 4 f$ and $4 d \rightarrow n p(n=5,6)$ autoionizing excitations from the ${ }^{2} F_{5 / 2}$ metastable state, although specific spectroscopic assignments were not made. A possible small step in the photoionization cross section near $136.8 \mathrm{eV}$ might

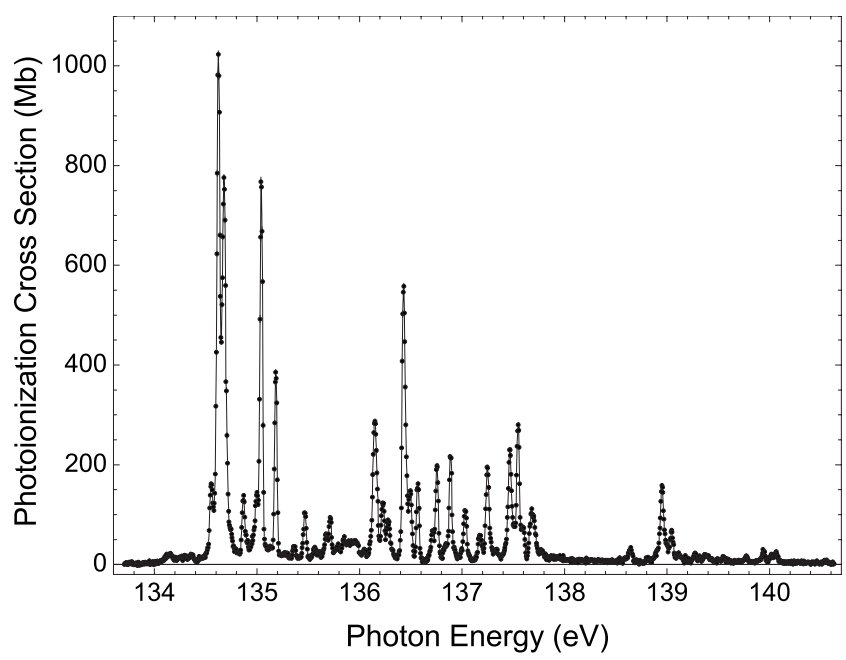

FIG. 9. Single-photoionization cross section of $\mathrm{Ce}^{9+}$ at a photon energy resolution of $0.05 \mathrm{eV}$.

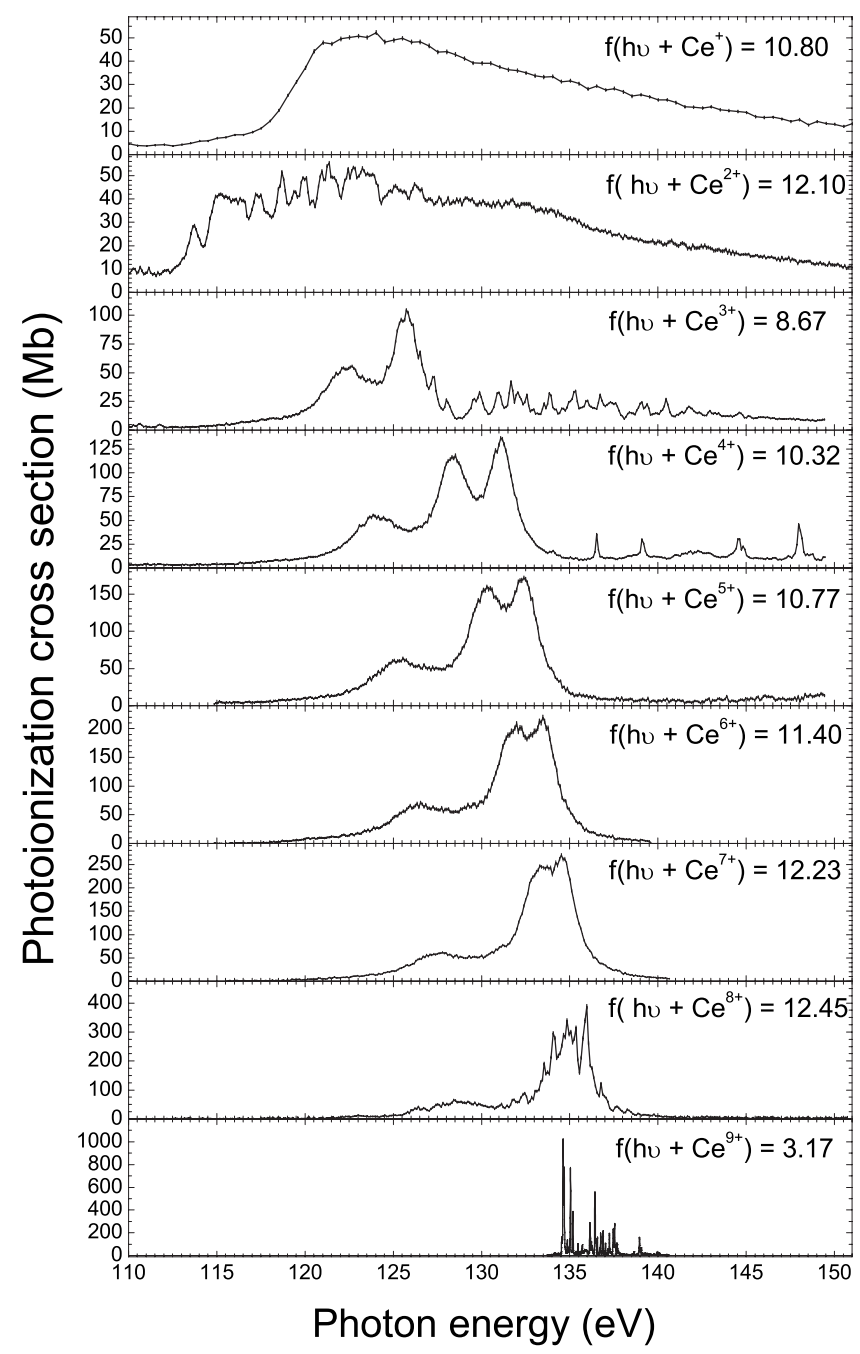

FIG. 10. Total photoionization cross sections for the cerium isonuclear sequence from $\mathrm{Ce}^{+}$through $\mathrm{Ce}^{9+}$ with their total measured oscillator strengths $f$. Double and triple photoionization are included in the total cross sections for those charge states for which they are energetically allowed in this energy range.

be due to the onset of direct photoionization of the $4 d^{10} 5 s^{2} 5 p^{1}{ }^{2} P_{3 / 2}$ metastable state, for which the calculated ionization potential is $136.82 \mathrm{eV}$ [18]. The resonance features above $136.8 \mathrm{eV}$ are assigned to autoionizing excitations of $4 d$ electrons to $4 f, 5 p$, and $6 p$ from the $4 d^{10} 5 s^{2} 5 p^{12} P_{3 / 2}$ metastable state. However, spectroscopic assignments of individual resonance features using the online version of the Cowan code were not possible.

Integration of the photoionization cross section of $\mathrm{Ce}^{9+}$ in the energy range of 133.7-140.6 eV yields an oscillator strength of 3.17. Since the ionization threshold of the ground state is close to $140.83 \mathrm{eV}$, the measured photoionization cross section in this energy range originates exclusively from the metastable states. Thus their proportion in the parent ion beam is estimated to be approximately $1 / 3$.

\section{SUMMARY AND CONCLUSIONS}

Photoionization cross sections for ions of the cerium isonuclear sequence from $\mathrm{Ce}^{+}$through $\mathrm{Ce}^{9+}$ were investigated 
by an ion-photon merged-beam technique using monochromatized synchrotron radiation. Competition between continuum and discrete electronic transitions from the filled $4 d$ subshell was observed along this ionic sequence, supporting the theoretical predictions for the collapse of the $n f$ orbitals with increasing initial ion charge. Metastable states were present in all the parent ion beams, which complicated the analysis of the measured cross sections. Fractions of the primary ion beams in metastable states were estimated to be $1 / 4$ for $\mathrm{Ce}^{4+}$ and $1 / 3$ for $\mathrm{Ce}^{9+}$. Figure 10 compares the total photoionization cross-section measurements for $\mathrm{Ce}^{+}$through $\mathrm{Ce}^{9+}$ ions and their associated oscillator strengths in the photon energy range of $4 d$ excitations. A broad asymmetric resonance dominates the cross section of $\mathrm{Ce}^{+}$, whereas the cross section of $\mathrm{Ce}^{2+}$ is characterized by strong resonances below the $4 d$ threshold and a delayed onset of direct photoionization. The $\mathrm{Ce}^{2+}$ cross section due to continuum excitation of the $4 d$ subshell decreases monotonically at high energies. Strong and broad features dominate the cross section of $\mathrm{Ce}^{3+}$, which also exhibits narrow but weaker resonances. The direct photoionization cross section of $\mathrm{Ce}^{3+}$ is much smaller than that for $\mathrm{Ce}^{+}$and $\mathrm{Ce}^{2+}$, indicating a growing competition between discrete and continuum photoionization processes with increasing ionization stage. Four Rydberg series were assigned in the single and double photoionization of $\mathrm{Ce}^{3+}$ arising from $4 d \rightarrow n f$ and $4 d \rightarrow n p$ transitions. In the $\mathrm{Ce}^{4+}$ cross section, the direct photoionization is almost negligible and only discrete transitions are observed, which is the case for all the higher charge states. That indicates the "collapse" of the $n f$ wave functions $[21,22]$ into the inner well of the Coulomb potential that binds the electrons of the $4 d$ subshell. This is a consequence of nuclear attraction dominating over the screening effect due to electron-electron interactions. In the case of $\mathrm{Ce}^{8+}$, strong narrow resonances arising from $4 d$ $\rightarrow 4 f$ autoionizing excitations dominate the photoionization cross section. Direct and indirect photoionization of the $5 \mathrm{~s}$ and $5 p$ subshells are also evident in the $\mathrm{Ce}^{6+}, \mathrm{Ce}^{7+}$, and $\mathrm{Ce}^{8+}$ cross sections, which account for the higher oscillator strengths in the experimental energy range for these ions. The $\mathrm{Ce}^{9+}$ cross section is dominated by more slowly autoionizing excitations of the $4 d$ electrons originating exclusively from the metastable states of this ion since $4 d$ excitation from the ground state populates only bound states.

\section{ACKNOWLEDGMENTS}

The authors are grateful to Dr. Ulyana Safronova for helpful discussions concerning the electronic structure of these ions and for identifications of metastable states. This research was supported by the U.S. Department of Energy, Division of Chemical Sciences, Biosciences, and Geosciences under Grant No. DOE-FG02-03ER15424.
[1] E. Biémont and P. Quinet, Phys. Scr. T105, 38 (2003).

[2] E. Biémont, P. Quinet, and T. A. Ryabchikova, Mon. Not. R. Astron. Soc. 336, 1155 (2002).

[3] C. R. Cowley, Astrophys. J., Suppl. Ser. 32, 631 (1976).

[4] T. B. Lucatorto, T. J. McIlrath, J. Sugar, and S. M. Younger, Phys. Rev. Lett. 47, 1124 (1981).

[5] J. M. Bizau, D. Cubaynes, J. M. Esteva, F. J. Wuilleumier, C. Blancard, J. Bruneau, J. P. Champeaux, A. Compant LaFontaine, C. Couillaud, R. Marmoret, C. Rémond, D. Hitz, M. Delaunay, N. Haque, P. C. Deshmukh, H. L. Zhou, and S. T. Manson, Phys. Rev. Lett. 87, 273002 (2001).

[6] J. M. Bizau, D. Cubaynes, M. Richter, F. J. Wuilleumier, J. Obert, J. C. Putaux, T. J. Morgan, E. Källne, S. Sorensen, and A. Damany, Phys. Rev. Lett. 67, 576 (1991).

[7] J. M. Bizau, D. Cubaynes, P. Gérard, and F. J. Wuilleumier, Phys. Rev. A 40, 3002 (1989).

[8] J. M. Bizau, J. M. Esteva, D. Cubaynes, F. J. Wuilleumier, C. Blancard, A. C. LaFontaine, C. Couillaud, J. Lachkar, R. Marmoret, C. Rémond, J. Bruneau, D. Hitz, P. Ludwig, and M. Delaunay, Phys. Rev. Lett. 84, 435 (2000).

[9] J. M. Bizau, C. Blancard, D. Cubaynes, F. Folkmann, J. P. Champeaux, J. L. Lemaire, and F. J. Wuilleumier, Phys. Rev. A 73, 022718 (2006).

[10] J. M. Bizau, E. Bouisset, C. Blancard, J. P. Champeaux, A. Compant la Fontaine, C. Couillaud, D. Cubaynes, D. Hitz, C. Vinsot, and F. J. Wuilleumier, Nucl. Instrum. Methods Phys. Res. B 205, 290 (2003).

[11] G. O'Sullivan, C. McGuinness, J. T. Costello, E. T. Kennedy, and B. Weinmann, Phys. Rev. A 53, 3211 (1996).
[12] A. Cummings, C. McGuinness, G. O’Sullivan, J. T. Costello, J. P. Mosnier, and E. T. Kennedy, Phys. Rev. A 63, 022702 (2001).

[13] A. Aguilar, J. D. Gillaspy, G. F. Gribakin, R. A. Phaneuf, M. F. Gharaibeh, M. G. Kozlov, J. D. Bozek, and A. L. D. Kilcoyne, Phys. Rev. A 73, 032717 (2006).

[14] A. Müller, S. Schippers, M. Habibi, D. Esteves, J. C. Wang, R. A. Phaneuf, A. L. D. Kilcoyne, A. Aguilar, and L. Dunsch, Phys. Rev. Lett. 101, 133001 (2008).

[15] A. M. Covington, A. Aguilar, I. R. Covington, M. F. Gharaibeh, G. Hinojosa, C. A. Shirley, R. A. Phaneuf, I. Álvarez, C. Cisneros, I. Dominguez-Lopez, M. M. Sant'Anna, A. S. Schlachter, B. M. McLaughlin, and A. Dalgarno, Phys. Rev. A 66, 062710 (2002).

[16] H. Kjeldsen, P. Andersen, F. Folkman, J. E. Hansen, M. Kitajima, and T. Andersen, J. Phys. B 35, 2845 (2002).

[17] Yu. Ralchenko, A. E. Kramida, J. Reader, and NIST ASD Team, NIST Atomic Spectra Database, Version 3.1.5, 2008, http://physics.nist.gov/PhysRefData/ASD/index.html

[18] R. D. Cowan, Cowan Atomic Structure Code, http:// aphysics2.lanl.gov/cgi-bin/ION/runlanl.pl

[19] R. D. Cowan, J. Opt. Soc. Am. 58, 808 (1968).

[20] R. D. Cowan, The Theory of Atomic Structure and Spectra (University of California Press, Berkeley, CA, 1981).

[21] K. T. Cheng and C. Froese Fischer, Phys. Rev. A 28, 2811 (1983).

[22] K. T. Cheng and W. R. Johnson, Phys. Rev. A 28, 2820 (1983). 